\title{
Nursing Education, Trauma and Care in the Intensive Care
}

\author{
Marianne Frieri ${ }^{1 *}$, Krishan Kumar ${ }^{2}$ and Anthony Boutin ${ }^{3}$ \\ ${ }^{1}$ Division of Allergy Immunology, Department of Medicine, Nassau University Medical Center, USA \\ ${ }^{2}$ Division of Pediatric, Department of Emergency Medicine, Nassau University Medical Center, USA \\ ${ }^{3}$ Division of Adult Emergency Medicine, Department of Emergency Medicine, Nassau University Medical Center, USA
}

*Corresponding author: Marianne Frieri, M.D, Ph.D, Division of Allergy Immunology, Department of Medicine, Nassau University Medical Center, 2201 Hempstead Turnpike, East Meadow, New York 11554, USA, Tel: 516-572-6501, Fax: 516 572-5609,E-mail:mfrieri@numc.edu

\begin{abstract}
Objective: The purpose of this article is to understand, nursing education, trauma and care in the intensive care especially related to nursing. Several methods and limitations of the studies were addressed.

Post operative cardio-thoracic surgical patients experiencing nursing bedside handover, a standardized infant positioning assessment tool and a bedside education program by registered nurses and highly specialized nurses in intensive care and their relation to patient outcome and nursing workload related to obese patients was described. In addition, head injury prevention, traumatic brain injury, a survey of nurses' perceptions in caring for patients with traumatic brain injury, outcomes and interventions for patients undergoing cardiac catheterization with nursing diagnosis of impaired tissue integrity, trauma informed care as a guiding philosophy and nursing practice and was reviewed. Immune function changes, which can lead to trauma stress management in trauma causing an immune imbalance in susceptible individuals, the neuroimmune system, oxidative stress were also addressed.
\end{abstract}

\section{Keywords}

Trauma, Nursing education, Outcomes, Care in the intensive care

\section{Introduction}

\section{Nursing education}

Patients with Traumatic Brain Injury (TBI) and their families rely on health care providers, particularly nurses, to provide accurate information, yet inaccurate beliefs about TBI have been found among nurses. Although prior studies have assessed nurses' beliefs about TBI recovery and rehabilitation, none have assessed specific beliefs about the nursing role to care for these patients. The purpose of this study was to investigate nurses' beliefs and learning preferences about caring for patients with moderate-to-severe TBI [1]. A cross-sectional survey was administered to 513 nurses at a Midwestern hospital between October and December 2014 (20.3\% response rate). Latent class analysis was used. Findings showed that nurses had inaccurate beliefs about TBI relating to recovery and the nursing role, and had significant differences in learning preferences. These findings have implications for development of educational and training interventions specific to nurses to ensure that they have factual information about TBI and to clarify the nursing role [1].

The purpose of this study was to determine nurses' perceptions about caring for patients with traumatic brain injury [2].

Annually, it is estimated that over 10 million people sustain a traumatic brain injury around the world. Patients with traumatic brain injury and their families are often concerned with expectations about recovery and seek information from nurses. Nurses' perceptions of care might influence information provided to patients and families, particularly if inaccurate knowledge and perceptions are held. Thus, nurses must be knowledgeable about care of these patients.

\section{Methods}

A cross-sectional survey, the Perceptions of Brain Injury Survey (PBIS), was completed electronically by 513 nurses between October and December 2014. Data

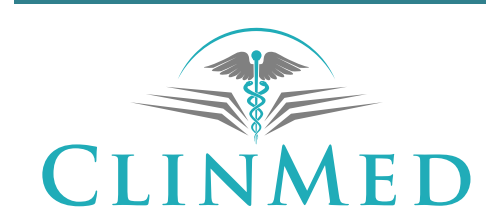

INTERNATIONAL LIBRARY 
were analysed with structural equation modelling, factor analysis, and pairwise comparisons.

Using latent class analysis, authors were able to divide nurses into three homogeneous sub-groups based on perceived knowledge: low, moderate and high. Findings showed that nurses who care for patients with traumatic brain injury the most have the highest perceived confidence but the lowest perceived knowledge. Nurses also had significant variations in training [2]. As there is limited literature on nurses' perceptions of caring for patients with traumatic brain injury, these findings have implications for training and educating nurses, including direction for development of nursing educational interventions.

\section{Relevance to Clinical Practice}

As the incidence of traumatic brain injury is growing, it is imperative that nurses be knowledgeable about care of patients with these injuries. The traumatic brain injury PBIS can be used to determine inaccurate perceptions about caring for patients with traumatic brain injury before educating and training nurses [2].

This paper described a nursing workforce practice development process to implement Trauma Informed Care as an inpatient model of mental health nursing care [3]. Trauma Informed Care is an evidence-based approach to care delivery that is applicable to mental health inpatient units; while there are differing strategies for implementation, there is scope for mental health nurses to take on Trauma Informed Care as a guiding philosophy, a model of care or a practice development project within all of their roles and settings in order to ensure that it has considered, relevant and meaningful implementation. The principles of Trauma Informed Care may also offer guidance for managing workforce stress and distress associated with practice change [3].

The purpose of this study was to describe the experiences of postoperative cardio-thoracic surgical patients experiencing nursing bedside handover [4]. A descriptive qualitative approach was undertaken. A purposeful sampling technique was adopted, including 14 patients who went through cardio-thoracic surgery and witnessed at least two bedside handovers. The study was performed in a Cardio-thoracic ICU localized in a Joint Commission International accredited Academic Hospital in north-eastern Italy from August to November 2014. The experience of patients participating at the bedside handover is based on four main themes: (1) 'Discovering a new nursing i5dentity', (2) 'Being apparently engaged in a bedside handover', (3) 'Experiencing the paradox of confidentiality' and (4) 'Having the situation under control' [4]. With the handover performed at the bedside in a postoperative setting, two interconnected potential effects may be achieved with regard to patients, nurses and the nursing profession. Nurses have a great oppor- tunity to express their closeness to patients and to promote awareness of the important growth that nursing has achieved over the years as a profession and discipline [4]. Therefore, patients may better perceive nursing competence and feel safer during the postoperative care pathway. They can appreciate nurses' humanity in caring and trust their competence and professionalism.

\section{Trauma}

A common type of pediatric trauma, can lead to adverse psychosocial outcomes, including posttraumatic stress disorder. A recent paper reviewed evidence-based parent programs to support children hospitalized after a traumatic injury [5]. The authors used qualitative methods in evaluation and intervention and completed a formative research study to develop a new program of psychological first aid and held focus groups with parents and pediatric trauma providers of children hospitalized at a Level I Children's Hospital because of an injury in 2012. They asked focus group participants to describe reactions to trauma and review drafts of our intervention materials They linked injured kids, and educated parents in supporting their children after a severe traumatic injury [5]. In their results health professionals and caregivers reported a broad spectrum of emotional responses by their children or patients. Difficulties were experienced during recovery at home and upon returning to school. All parents and health professionals recommended that interventions be offered to parents either in the emergency department or close to discharge among admissions. Thus, results from this study strongly indicated a need for post trauma interventions, particularly in rural settings, to support families of children to address the psychosocial outcomes in the aftermath of an injury. Findings described the process of intervention development that responds to the needs of an affected population [5].

The effects of exposure to childhood trauma may be transmitted across generations and, the time period(s) and mechanism(s) have yet to be clarified. These authors addressed the hypothesis that intergenerational transmission may begin during intrauterine life via the effect of maternal childhood trauma exposure on placental-fetal stress physiology, specifically placental Corticotropin Releasing Hormone pCRH [6].

The study was conducted in a socio-demographically diverse cohort of 295 pregnant women. Childhood trauma exposure was assessed using the Childhood Trauma Questionnaire. Placental CRH concentrations were quantified in maternal blood collected serially over the course of gestation. Linear mixed effects and Bayesian piece-wise linear models were employed to test hypothesized relationships. Maternal Childhood Trauma (CT+) was significantly associated with $\mathrm{pCRH}$ production. Compared with nonexposed women, CT+ was associated with an almost $25 \%$ increase in $\mathrm{pCRH}$ toward the end of gestation, and the pCRH trajectory of CT+ women exhibited an approximately twofold steeper increase after the $\mathrm{pCRH}$ inflection point at 19 weeks gestation. The authors concluded this finding represents the first 
report linking maternal child hood trauma-exposure with placental-fetal stress physiology, thus identifying a potential novel biological pathway of intergenerational transmission that may operate as early as during intrauterine life [7]. Blunt childhood renal trauma with pre-existing renal abnormalities was reviewed [8]. The retroperitoneal location affords the kidneys some protection from the forces experienced in blunt abdominal trauma, however the kidneys are at greater risk of injury when a disease process exposes them from their normal shielded location [8]. The injuries may appear to be disproportionate in relation to the severity of the trauma history, confusing the imaging findings, and recognition of both the underlying disease process as well as the manifestations of acute trauma is important [8].

A recent study compared the test characteristics of clinician suspicion with a derived clinical prediction rule to identify children at risk of intra-abdominal injuries undergoing acute intervention following blunt torso trauma [9]. This was a planned subanalysis of a prospective, multicenter observational study of children (<18-years-old) with blunt torso trauma conducted in 20 EDs in the Pediatric Emergency Care Applied Research Network (PECARN) [9]. Clinicians documented their suspicion for the presence of intra-abdominal injuries needing acute intervention. The derived clinical prediction rule had a significantly higher sensitivity, but lower specificity, than clinician suspicion for identifying children with intra-abdominal injuries undergoing acute intervention. The higher specificity of clinician suspicion, however, did not translate into clinical practice, as clinicians frequently obtained abdominal CT scans in patients they considered very low risk. If validated, this prediction rule can assist in clinical decision-making around abdominal CT use in children with blunt torso trauma [9].

A toddler with a closed head injury six days prior to admission, recently diagnosed with post-concussive syndrome and acute otitis media presented to the emergency department with complaint of uncontrollable shaking of the head and extremities was discussed. Physical examination demonstrated dancing movements of the eyes and truncal ataxia, concerning for the diagnosis of opsoclonus-myoclonus syndrome. Magnetic resonance imaging study was consistent with a retroperitoneal mass that was confirmed as neuroblastoma by metaiodobenzylguanidine scan and later surgical resection. The authors presented this case, accompanied by a video of the patient, to help the emergency physician recognize this rare and often misdiagnosed syndrome [10].

The incidence of Venous Thrombo Embolism (VTE) in pediatric trauma patients is increasing. Consequences of VTE in children are significant. Studies have demonstrated increasing VTE risk in older pediatric trauma patients and improved VTE rates with institutional interventions [11]. A recent study developed a simple clinical tool to predict the risk of developing VTE in pediatric trauma patients based on a model created using a large national database and was internally validated [11]. The authors developed a simple clinical tool to predict the risk of developing VTE in pediatric trauma patients, based on a model created using a large national database and was internally validated. The clinical tool required external validation and provided an initial step toward the development of the specific VTE protocols for pediatric trauma patients [11].

A recent study compared the test characteristics of clinician suspicion with a derived clinical prediction rule to identify children at risk of intra-abdominal injuries undergoing acute intervention following blunt torso trauma [12]. This was a planned subanalysis of a prospective, multicenter observational study of children (< 18-years-old) with blunt torso trauma conducted in 20 EDs in the Pediatric Emergency Care Applied Research Network (PECARN) [12]. Clinicians documented their suspicion for the presence of intra-abdominal injuries needing acute intervention. The derived clinical prediction rule had a significantly higher sensitivity, but lower specificity, than clinician suspicion for identifying children with intra-abdominal injuries undergoing acute intervention. The higher specificity of clinician suspicion, however, did not translate into clinical practice, as clinicians frequently obtained abdominal CT scans in patients they considered very low risk. If validated, this prediction rule can assist in clinical decision-making around abdominal CT use in children with blunt torso trauma [12].

A well described consequence of traumatic injury is immune dysregulation, when an initial increase in immune activity is followed by a period of immune depression, leaving hospitalized trauma patients at an increased risk of nosocomial infections [12]. These authors discussed the emerging role of the neutrophil, the most abundant leukocyte in human circulation and the first line of defense against microbial challenge, in the initiation and propagation of the inflammatory response to trauma [12]. They reviewed the findings of the most recent studies to have investigated the impact of trauma on neutrophil function and discussed how alterations in neutrophil biology are being investigated as potential biomarkers to predict the outcome of hospitalized trauma patients [12]. With trauma-induced changes in neutrophil biology linked to the development of such post-traumatic complications as multiple organ failure and acute respiratory distress syndrome, these authors highlighted an area of research within the field of trauma immunology that is gaining considerable interest: the manipulation of neutrophil function as a means by which to potentially improve patient outcome [12].

The first phase, in addition to the injury by trauma, organ damage is caused by the immune system during a systemic inflammatory response, In the second phase the patient is more susceptible for sepsis due to host defense failure or immune paralysis [13]. The innate immune system is an immune monitor and has a very 
prominent role in organ failure after trauma. Polymorphonuclear phagocytes and monocytes are the main effector-cells of the innate immune system that are involved in organ failure and are controlled by cytokines, chemokines, complement factors and specific tissue signals [13].

Despite the enormous efforts to clarify mechanisms of the development of Multiple Organ Failure (MOF) following trauma, MOF following trauma is still a leading cause of late post-injury death and morbidity [14]. It has been proven that excessive systemic inflammation following trauma participates in the development of MOF. The inflammatory response is a host-defense response; however, on occasion, this response turns around to cause deterioration to host depending on exo- and endogenic factors. This review described the pathophysiological approach for MOF after trauma studied so far and also introduced the prospects of this issue for the future [14].

Following major trauma, IL- 6 release correlates with injury severity, complications, and mortality [15]. Interleukin-10 (IL-10) can markedly inhibit lymphocyte and phagocytic functions, essential for an adequate immune response [16]. In this early study patients who died from injury or developed posttraumatic complications due to multiple organ dysfunction syndrome had elevated IL10 levels in comparison with injured patients with an uneventful posttraumatic course [16]. Thus, trauma causes an enhanced release of IL-10 dependent on the severity of injury and because increased IL-10 levels are significantly related to posttraumatic complications, IL10 may be involved in their pathogenesis [16]. A significant increase of both IL- 6 and IL-10 concentrations was found over time in a reported study, with a significant correlation between the Injury Severity Score and the levels of both IL- 6 and IL-10 at all sampling points [16]. Serum concentrations of IL- 6 and IL- 10 were significantly higher in patients not surviving 30 days. The early systemic inflammatory response measured as IL- 6 and IL10 in serum is correlated with injury severity and 30-day mortality following trauma [16].

\section{IL-6, Obesity and Trauma}

Severe asthma is a complex heterogeneous disease associated with older age and obesity. The presence of eosinophilic (type 2) inflammation in some but not all patients with severe asthma predicts responsiveness to current treatments, but new treatment approaches will require a better understanding of non-type 2 mechanisms of severe asthma [17]. These authors considered the possibility that systemic inflammation, which arises in subgroups of obese and older patients, increases the severity of asthma. Interleukin-6 (IL-6) is a biomarker of systemic inflammation and metabolic dysfunction, and we aimed to explore the association between IL- 6 concentrations, metabolic dysfunction, and asthma severity [17].
Trauma is a leading cause of death in both military and civilian populations worldwide [18]. Although medical advances have improved the overall morbidity and mortality often associated with trauma, additional research and innovative advancements in therapeutic interventions are needed to optimize patient outcomes. Cell-based therapies present a novel opportunity to improve trauma and critical care at both the acute and chronic phases that often follow injury. Although this field is still in its infancy, animal and human studies suggest that stem cells may hold great promise for the treatment of brain and spinal cord injuries, organ injuries, and extremity injuries such as those caused by orthopedic trauma, burns, and critical limb ischemia [19]. Barriers in the translation of cell therapies that include regulatory obstacles, challenges in manufacturing and clinical trial design, and a lack of funding are critical areas in need of development. In 2015, the Department of Defense Combat Casualty Care Research Program held a joint military-civilian meeting as part of its effort to inform the research community about this field and allow for effective planning and programmatic decisions regarding research and development. The objective of this article was to provide a "state of the science" review regarding cellular therapies in trauma and critical care, and to provide a foundation from which the potential of this emerging field can be harnessed to mitigate outcomes in critically ill trauma patients [19].

The types of splenic traumas, accompanying injuries management and results in children in 90 patients (64 boys, 26 girls) treated for splenic injuries as a result of blunt abdominal trauma between 2005-2012 were assessed [20]. Causes of trauma were falls from height, pedestrian traffic accidents passenger traffic accidents, bicycle accidents and falling objects from height. Splenic injury alone was observed in 57 patients $(63.3 \%)$ and other organ injuries together with splenic injury in 33 patients (36.7\%). Splenectomy was performed in six patients (6.6\%) due to hemodynamic instability and small intestine repair due to small intestine injury in one patient (1.1\%). None of these patients died from their injuries. The authors stated a large proportion of splenic injuries recover with conservative therapy and some of the advantages of conservative therapy includes short hospitalization time, less need for blood transfusion, and less morbidity and mortality. Falls from height and traffic accidents are important factors in etiology. The possibility of other organ injuries together with splenic injuries should be considered [20].

A integrative literature review of the current literature evaluating trauma nursing education was conducted [21]. A variety of trauma nursing courses exist, to educate nurses working in trauma settings, and to maintain their continuing professional development. Despite an increase in the number of courses delivered, there appears to be a lack of evidence to demonstrate the 
effectiveness of trauma nursing education and in particular the justification for this resource allocation [21]. A search of international literature on trauma nursing education evaluation published in English from 1985 to 2015 was conducted through electronic databases CINAHL Plus, Google Scholar, PubMed, Austhealth, Science Citation Index Expanded (Web of Science), Sciverse Science Direct (Elsevier) \& One file (Gale). Only peer reviewed journal articles identifying trauma course and trauma nursing course evaluation have been included in the selection criteria. An integrative review of both quantitative and qualitative literature guided by Whittemore and Knafl's theoretical framework using Bowling's and Pearson's validated appraisal checklists, was conducted for three months. Only 17 studies met the inclusion criteria, including 14 on trauma course evaluation and 3 on trauma nursing course evaluation. The study findings were presented as two main themes: the historical evolution of trauma nursing education and evaluation of trauma nursing education outcomes [21]. Trauma nursing remains in its infancy and education in this specialty is mainly led by continuing professional development courses. The shortage of evaluation studies on trauma nursing courses reflects the similar status in continuing professional development course evaluation. A trauma nursing course evaluation study should address the gap in this under researched area [21].

This article summarized the successes and future implications for a nurse practitioner-driven committee on process improvement in trauma. The trauma nurse practitioner is uniquely positioned to recognize the need for clinical process improvement and enact change within the clinical setting [22]. Application of the Strong Model of Advanced Practice proves to actively engage the trauma nurse practitioner in process improvement initiatives. Through enhancing nurse practitioner professional engagement, the committee aims to improve health care delivery to the traumatically injured patient [22]. A retrospective review of the committee's first year reveals trauma nurse practitioner success in the domains of direct comprehensive care, support of systems, education, and leadership. The need for increased trauma nurse practitioner involvement has been identified for the domains of research and publication [22].

\section{Conclusion}

This article reviewed the understand nursing education, trauma and care in the intensive care especially related to nursing Post operative cardio-thoracic surgical patients experiencing nursing bedside handover, a standardized infant positioning assessment tool and a bedside education program by registered nurses and highly specialized nurses in intensive care and their relation to patient outcome and nursing workload related to obese patients was described. In addition, head injury prevention, traumatic brain injury, a survey of nurses' perceptions in caring for patients with traumat- ic brain injury, outcomes and interventions for patients undergoing cardiac catheterization with nursing diagnosis of impaired tissue integrity, trauma informed care as a guiding philosophy and nursing practice and was reviewed. Immune function changes, which can lead to trauma stress management in trauma causing an immune imbalance in susceptible individuals, the neuroimmune system, oxidative stress were also addressed. Various methods and limitations were also addressed.

\section{References}

1. Oyesanya TO, Thomas MA, Brown RL, Turkstra LS (2016) Nurses' Beliefs about Caring for Patients with Traumatic Brain Injury. West J Nurs Res 38: 1114-1138.

2. Oyesanya TO, Brown RL, Turkstra LS (2017) Caring for patients with traumatic brain injury: a survey of nurses' perceptions. J Clin Nurs 26: 1562-1574.

3. Isobel S, Edwards C (2017) Using trauma informed care as a nursing model of care in an acute inpatient mental health unit: A practice development process. Int $\mathrm{J}$ Ment Health Nurs 26: 88-94.

4. Lupieri G, Creatti C, Palese A (2016) Cardio-thoracic surgical patients' experience on bedside nursing handovers: Findings from a qualitative study. Intensive Crit Care Nurs 35: 28-37.

5. Ramirez $M$, Toussaint $M$, Woods $B$, Harland $K$, Wetjen K, et al. (2015) Link for Injured Kids: A Patient-Centered Program of Psychological First Aid After Trauma. Pediatr Emerg Care.

6. Moog NK, Buss C, Entringer S, Shahbaba B, Gillen DL, et al. (2016) Maternal exposure to childhood trauma is associated during pregnancy with placental-fetal stress physiology. Biol Psychiatry 79: 831-839.

7. Dahlstrom K, Dunoski B, Zerin JM (2015) Blunt renal trauma in children with pre-existing renal abnormalities. Pediatr Radiol 45: 118-123.

8. Hang B, Babcock L, Hornung R, Ho M, Pomerantz WJ (2015) Can Computerized Neuropsychological Testing in the Emergency Department Predict Recovery for Young Athletes With Concussions? Pediatr Emerg Care 31: 688-693.

9. Mahajan P, Kuppermann N, Tunik M, Yen K, Atabaki SM, et al. (2015) Comparison of Clinician Suspicion Versus a Clinical Prediction Rule in Identifying Children at Risk for Intra-abdominal Injuries After Blunt Torso Trauma. Acad Emerg Med 22: 1034-1041.

10. Wu H, Mody AP (2015) A Toddler with Uncontrollable Shaking after a Minor Fall. Pediatr Emerg Care.

11. Connelly CR, Laird A, Barton JS, Fischer PE, Krishnaswami S, et al. (2015) A clinical tool for the prediction of venous thromboembolism in pediatric trauma patients. JAMA Surg 151: $50-57$

12. Hazeldine J, Hampson P, Lord JM (2014) The impact of trauma on neutrophil function. Injury 45: 1824-1833.

13. Hietbrink F, Koenderman L, Rijkers G, Leenen L (2006) Trauma: the role of the innate immune system. World $\mathrm{J}$ Emerg Surg 1: 15.

14. Tsukamoto T, Chanthaphavong RS, Pape HC (2010) Current theories on the pathophysiology of multiple organ failure after trauma. Injury 41: 21-26.

15. Neidhardt R, Keel M, Steckholzer U, Safret A, Ungethuem $U$, et al. (1997) Relationship of interleukin-10 plasma levels 
to severity of injury and clinical outcome in injured patients. J Trauma 42: 863-870.

16. Stensballe J, Christiansen M, Tønnesen E, Espersen K, Lippert FK, et al. (2009) The early IL-6 and IL-10 response in trauma is correlated with injury severity and mortality. Acta Anaesthesiol Scand 53: 515-521.

17. Peters MC, McGrath KW, Hawkins GA, Hastie AT, Levy BD, et al. (2016) Plasma interleukin-6 concentrations, metabolic dysfunction, and asthma severity: a cross-sectional analysis of two cohorts. Lancet Respir Med 4: 574-584.

18. Pati S, Pilia M, Grimsley JM, Karanikas AT, Oyeniyi B, et al. (2015) Cellular Therapies in Trauma and Critical Care Medicine: Forging New Frontiers. Shock 44: 505-523.

19. Tulloch LG, Chan JD, Carlbom DJ, Kelly MJ, Dellit TH, et al. (2017) Epidemiology and Microbiology of Sepsis Syndromes in a University-Affiliated Urban Teaching Hospital and Level-1 Trauma and Burn Center. J Intensive Care Med 32: 264-272.

20. Arslan S, Guzel M, Turan C, Doğanay S, Kopru M (2015) Management and treatment of splenic trauma in children. Ann Ital Chir 86: 30-34.

21. Ding M, Metcalfe H, Gallagher O, Hamdorf JM (2016) Evaluating trauma nursing education: An integrative literature review. Nurse Educ Today 44: 33-42.

22. West SK (2016) Advanced Practice Nursing Committee on Process Improvement in Trauma: An Innovative Application of the Strong Model. J Trauma Nurs 23: 227-230. 